Check for updates

Cite this: RSC Adv., 2019, 9, 301

Received 2nd October 2018

Accepted 2nd December 2018

DOI: $10.1039 / \mathrm{c} 8 \mathrm{ra0} 8163 \mathrm{~h}$

rsc.li/rsc-advances

\title{
A targeted biocompatible organic nanoprobe for photoacoustic and near-infrared-II fluorescence imaging in living mice $\uparrow$
}

\author{
Xinhui Xie, ${ }^{\star a}$ Yili Hu, ${ }^{a}$ Chao Zhang, (D) ${ }^{b}$ Jialei Song, ${ }^{a}$ Suyang Zhuang ${ }^{\mathrm{a}}$ \\ and Yuntao Wang ${ }^{a}$
}

\begin{abstract}
Multimodal molecular imaging probes have attracted much attention, and they possess great potential to accurately diagnose diseases due to the synergistic superiorities of multiple complementary imaging. Herein, a targeted biocompatible organic nanoplatform (IR-PEG-FA) with a strong optical absorption in the near-infrared window (NIR-I) for photoacoustic imaging (PAI) and excellent second near-infrared (NIR-II) fluorescence imaging property for NIR-II imaging is fabricated. The dual-modal nanoprobe is composed of the small organic dye molecule IR-1061, water-soluble poly(ethylene glycol) (PEG) and folic acid (FA) as the targeted ligands. Depending on the strength of high temporal resolution and preeminent spatial resolution, the targeted biocompatible dual-mode nanoprobe for PAI and NIR-II imaging can provide more detailed date of cancers and diseases, and enables us to specifically diagnose them through quite a precise way.
\end{abstract}

\section{Introduction}

The territory of bio-imaging and cancer diagnosis has witnessed a great increase in the past decades, mainly owing to the rapid progress of numerous imaging modalities with eminent sensitivity and resolution. ${ }^{1-4}$ These emerging imaging modalities possessed tremendous promise for remarkably perfecting patient medical treatment and potentially reducing damage to the normal tissue..$^{5-7}$ Over the past years, the most noteworthy evolution is the introduction of multimodality molecular imaging, which is regarded as the combination of several imaging modalities for the sake of maximizing their respective advantages while conquering the defects of each modality..$^{8-12}$ Thus far, plenty of multimodality imaging platforms have been successfully adopted to improve disease diagnostic accuracy and efficacy. ${ }^{13-20}$

Photoacoustic imaging (PAI), which combines optical signal detection with ultrasonic information collection, possesses noticeable preponderance for biological imaging with a relatively favorable tissue penetration and spatial resolution. ${ }^{21-28}$ However, to realize more higher resolution, the tissue penetration of PAI under the first near-infrared region (NIR-I, 650$950 \mathrm{~nm}$ ) is still restricted owing to the optical attenuation. ${ }^{29}$ To

${ }^{a}$ The Department of Orthopedics, Zhong Da Hospital, School of Medicine, Southeast University, Nanjing, Jiangsu, 210009, China. E-mail: xiexinghuixxh@163.com ${ }^{b}$ Collaborative Innovation Center of Chemistry for Life Sciences, College of Engineering and Applied Sciences, Nanjing University, Nanjing, Jiangsu, 210093, China

$\dagger$ Electronic supplementary information (ESI) available. See DOI: $10.1039 / \mathrm{c} 8 \mathrm{ra} 08163 \mathrm{~h}$ address the limitation, the developing imaging agents with fluorescence emission in the second near-infrared (NIR-II) region (1000-1700 $\mathrm{nm})$ have incomparable advantages. ${ }^{30}$ These NIR-II agents show more deeper tissue penetration and higher spatial resolution, which attributes to the reduced autofluorescence and diminished optical scattering. ${ }^{31-36} \mathrm{~A}$ recent successful paradigm in the NIR-II window for in vivo lymphatic, ${ }^{30,35}$ microvascular, ${ }^{32,37}$ and tumor imaging ${ }^{38,39}$ repeatedly demonstrated the superior temporal and spatial resolutions of the technique. Particularly, NIR-II imaging can accurately detect tumor margins based on real-time images to perfect diagnostic accuracy and further guarantee the triumph of tumor resection. ${ }^{34}$ On account of the dominant superiority over conventional NIR-I imaging, NIR-II imaging possesses great opportunities in preclinical applications and clinical translation.$^{40}$ Hence, in view of the feasibility and compatibility of PAI and NIR-II fluorescence imaging, it promotes us to integrate these two imaging modalities together to obtain a synergetic and complementary information to enhance the efficacy of cancer diagnosis.

Thus far, inorganic nanomaterials, including single-walled carbon nanotubes, ${ }^{41,42}$ quantum dots, ${ }^{43,44}$ and rare-earth nanoparticles, ${ }^{18,45}$ have been employed for the in vivo NIR-II imaging. Owing to undiscovered long-term toxicity misgivings, it would urge us to explore organic molecule dyes for in vivo imaging to promote the clinical translation. ${ }^{46}$ More recently, some organic nanomaterials (small molecule and conjugated polymer) ${ }^{30,33,40,47}$ have been developed as attractive NIR-II agents. Particularly, Cheng et al. developed the first dual-mode probe composed of D-A-D-type chromophores, which exhibited excellent NIR-II 
imaging and PAI properties in living mice. ${ }^{\mathbf{4 8}}$ Despite the rapid progress in exploring NIR-II imaging agents, there are still rare reports about the dual-mode contrast agents based on PAI/NIRII imaging. Therefore, developing a novel biocompatible PAI/ NIR-II imaging agent will greatly promote the development of the field in molecule imaging and diagnosis.

Herein, we developed a targeted biocompatible dual-mode contrast agent IR-PEG-FA (Scheme 1), which is composed of IR-1061 (a commercial organic dye) and folic acid (FA), for in vivo PAI and NIR-II imaging (Scheme 2). The obtained IR-PEGFA exhibited a broad NIR absorption region with a peak at $\sim 810 \mathrm{~nm}$ and a maximum emission wavelength at $1100 \mathrm{~nm}$. Owing to the introduction of a molecular imaging ligand, along with the enhanced permeation and retention (EPR) effect of the pathological tumor sites, the IR-PEG-FA possessed more tumorspecific targeting performance for the in vivo dual-mode imaging. We believe that the tailor-made biocompatible dualmode contrast agent will provide a new strategy for dual-mode PAI and NIR-II imaging and light up the clinical applications in cancer diagnosis.

\section{Experimental section}

\section{Chemicals}

The functionalized poly(ethylene glycol) $\left(\mathrm{SH}-\mathrm{PEG}_{5000}-\mathrm{NH}_{2}\right)$ was purchased from ToYongBio Tech. Inc. (Shanghai, China). All the other reagents were commercially acquired (Sigma Aldrich) and used directly without further purification.

\section{Material characterization}

NMR spectra were recorded using a $400 \mathrm{MHz}$ spectrometer (Bruker Ultra Shield Plus). The absorption spectra were recorded using a UV-3600 spectrophotometer (Shimadzu). NIR fluorescence spectra were recorded using an Edinburgh FLSP920

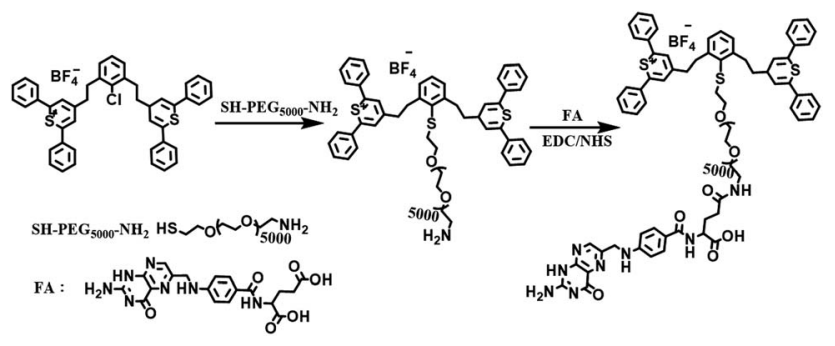

Scheme 1 Synthesis of IR-PEG-FA.

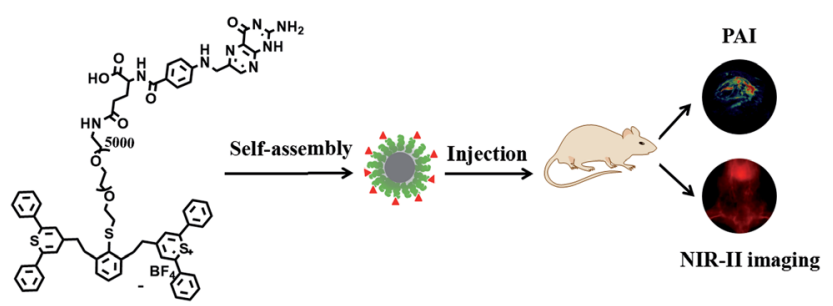

Scheme 2 Schematic description of the prepared IR-PEG-FA as the dual-modal imaging agent for PAI and NIR-II in living mice. fluorescence spectrophotometer in the 900-1500 $\mathrm{nm}$ region with an $808 \mathrm{~nm}$ laser excitation. The ALV/CSG-3 laser scattering spectrometer was used for dynamic light scattering (DLS) investigation. The HT7700 transmission electron microscope (TEM) was used for recording TEM images at an acceleration voltage of $100 \mathrm{kV}$. The Nexus-128 photoacoustic imaging (PAI) instrument (680-950 nm) was used for PAI in vitro and in vivo. The methyl thiazolyl tetrazolium (MTT) study was performed using a BioTek spectrophotometer (PowerWave XS/XS2 microplate). The in vivo NIR-II fluorescence imaging was performed using the home-built imaging set-up (CDD: NIRvana TE 640). The excitation wavelength was fixed at $808 \mathrm{~nm}$ (laser power: $\sim 100 \mathrm{~mW} \mathrm{~cm}^{-2}$ ).

\section{Synthesis of IR-PEG-FA}

SH-PEG ${ }_{5000}-\mathrm{NH}_{2}(200 \mathrm{mg})$ and IR-1061 $(50 \mathrm{mg})$ were dissolved in $30 \mathrm{~mL}$ dimethyl sulfoxide and allowed to react at room temperature for $36 \mathrm{~h} .{ }^{47}$ After removing the solvent by distillation under reduced pressure, the mixture was redissolved in pure water. To remove unreacted PEG, the acquired solution was further purified by dialysis with deionized water for $72 \mathrm{~h}$ using a dialysis bag (MW: $7000 \mathrm{Da}$ ). The obtained product was then dried via freeze-drying to acquire a brown compound (IR-PEG). Then, $100 \mathrm{mg}$ IR-PEG, NHS (25 mg), EDC·HCl (40 mg), and $30 \mathrm{mg}$ folic acid (FA) were dissolved in $30 \mathrm{~mL}$ of dry $N, N$ dimethyl formamide (DMF) at room temperature. After $72 \mathrm{~h}$ of reaction, DMF was distilled by reduced pressure distillation, and the residual solid was dissolved in appropriate amounts of deionized water. To remove unreacted FA and other impurities, the obtained solution was further subjected to dialysis with deionized water for $72 \mathrm{~h}$ using a dialysis bag (MW: $7000 \mathrm{Da}$ ). The final product (IR-PEG-FA) was further dried by freeze-drying to yield a brown product.

\section{NIR fluorescence spectroscopy}

NIR fluorescence emission spectra were recorded using a spectrophotometer (Edinburgh FLSP920, 900-1500 nm) under laser excitation at $808 \mathrm{~nm}$. The excitation light at $808 \mathrm{~nm}$ was provided by a diode laser (Ti:sapphire system lasers, $808 \mathrm{~nm}$, $180 \mathrm{~mW}$ ) and filtered through a $900 \mathrm{~nm}$ long-pass filter. The excitation laser beam was passed through the sample of IR-PEGFA in PBS solutions $(\mathrm{pH}=7.4)$ and the emission signal was obtained with the transmission geometry. In order to reject the $808 \mathrm{~nm}$ laser light, an additional $900 \mathrm{~nm}$ long-pass filter was used to reduce the background interference.

\section{Preparation of IR-PEG-FA nanoparticles}

IR-PEG-FA nanoparticles were synthesized by directly dissolving IR-PEG-FA in phosphate-buffered saline (PBS, $\mathrm{pH}=7.4$ ) with continuous sonication. The gained NPs were then filtrated using a millipore filter $(0.22 \mu \mathrm{m})$ to remove the insoluble product or other impurities for cell and biological experiments.

\section{Cell viability assay}

The cytotoxicity of IR-PEG-FA was studied by an MTT experiment using U87MG and NIH-3T3 cells. These cells $\left(1 \times 10^{4}\right.$ cells 
per $\mathrm{mL}$ ) were cultured in 96-well plates. After 1 day of incubation, the medium was removed and a series of concentrations of IR-PEG-FA solution $\left(80,60,40,20\right.$, and $\left.10 \mu \mathrm{g} \mathrm{mL}^{-1}\right)$ were added into the well and cultured for another 1 day. After that, the cells were washed twice with saline, and MTT solution $(100 \mu \mathrm{L}$, $0.5 \mathrm{mg} \mathrm{mL}^{-1}$ ) was added into each well. After cultivation for $4 \mathrm{~h}$, the MTT solution was removed and $200 \mu \mathrm{L}$ of dimethylsulfoxide was added into each well and left for another $20 \mathrm{~min}$. A microplate reader was used for acquiring absorbance values at $490 \mathrm{~nm}$. The untreated cells were used as the control group and the cellular viability was set at $100 \%$.

\section{Tumor mouse model}

All U87MG tumor-bearing mice were treated on the basis of the rules set by the Animal Centre of Southeast University (Nanjing, China) and the experiments were approved by the Animal Ethics Committee of Model Animal Research Center of Nanjing University. To acquire the suitable U87MG tumor model for in vivo imaging, 6 week old mice were used. In addition, $1.5 \times 10^{6}$ U87MG cells were injected subcutaneously into the target location of the mice. The tumor-bearing mice can be adopted for in vivo imaging until the tumor grew up to approximately $8 \mathrm{~mm}$ in size.

\section{In vitro and in vivo photoacoustic imaging}

The in vitro PAI properties of IR-PEG-FA aqueous solutions (50, $100,200,400$, and $800 \mu \mathrm{g} \mathrm{mL}^{-1}$ ) were studied and the PA signals were recorded using the Endra PA tomography system at an excitation wavelength of $808 \mathrm{~nm}$. The gained images were further analyzed using the Image software, and an identical region-of-interest was performed for collecting the quantitative PA signal intensity. For in vivo PAI, the living mice were anesthetized using $1.5 \%$ isoflurane in flowing air, and they were administered with IR-PEG-FA $\left(150 \mu \mathrm{L}, 1 \mathrm{mg} \mathrm{mL} \mathrm{mL}^{-1}\right)$ through the tail vein. Then, the mice were transferred into a chamber where the temperature was set at $38^{\circ} \mathrm{C}$, and were provided with water for drinking. The Endra PA imaging system was utilized to collect the PA images at $808 \mathrm{~nm}$.

\section{In vitro and in vivo NIR-II imaging}

A $500 \mu \mathrm{L}$ polymerase chain reaction (PCR) tube with IR-PEG-FA aqueous solution was placed in the NIR-II imaging instrument, and the NIR-II fluorescence images were recorded. The final image was analyzed using the Image s software. To further detect the in vivo NIR-II imaging, the U87MG tumor-bearing living mice were intravenously injected through the tail with a $150 \mu \mathrm{L}$ solution of IR-PEG-FA $\left(1 \mathrm{mg} \mathrm{mL}{ }^{-1}\right)$ for the video-rate in vivo fluorescence imaging. The in vivo NIR-II fluorescence imaging was recorded using the home-built imaging set-up (CDD: NIRvana TE 640). The $808 \mathrm{~nm}$ laser excitation power was fixed at $\sim 100 \mathrm{~mW} \mathrm{~cm}^{-1}$ on the animal surface.

\section{Results and discussion}

\section{Preparation and characterisation of IR-PEG-FA}

The synthetic route of IR-PEG-FA is illustrated in Scheme 1. Briefly, aminated IR-PEG was first synthesized by introducing a functionalized poly(ethylene glycol) (PEG, $\left.M_{\mathrm{w}}=5000\right)$ chain (SH-PEG-NH ${ }_{2}$ ) into the IR1061 molecule. Then, the obtained amino group of IR-PEG can provide modifiable sites for the introduction of folic acid (FA) by the amidation reaction, affording the end-product, IR-PEG-FA (Fig. 1A). The successful linkage of PEG and FA can be determined from the MALDI-TOFMS spectra (Fig. S1 $\dagger$ ) and ${ }^{1} \mathrm{H}$ NMR spectra (Fig. S2 and S3†) of IR-PEG-FA. Particularly, the mass spectrum (Fig. S1 $\dagger$ ) exhibited two peaks with maximum molecular weight at 5806.621 Da and 6247.476 Da, which were attributed to the unsuccessful conjugation of a part of FA molecules. Owing to the hydrophobichydrophilic groups, IR-PEG-FA was directly dispersed in the phosphate-buffered saline ( $\mathrm{PBS}, \mathrm{pH}=7.4$ ) and could be spontaneously self-assembled to form nanoparticles, which gave brown color to the solution (Fig. 1B). Dynamic light scattering (DLS) showed that the IR-PEG-FA has similar average diameters of $\sim 77 \mathrm{~nm}$ (Fig. 1D). A spherical morphology was observed in the transmission electron microscopic (TEM) image with a particle size of $62 \pm 4.6 \mathrm{~nm}$ (Fig. 1C). The acquired bigger nanoparticle size of IR-PEG-FA in the DLS image could be attributed to the import of the PEG chains and its extension in an aqueous state. Besides, no significant insoluble substances and precipitation in the IR-PEG-FA solution were observed after storage in a refrigerator $\left(4^{\circ} \mathrm{C}\right)$ for 30 days (Fig. 2A), demonstrating its excellent stability in the aqueous solution. The optical performance of IR-PEG-FA was studied in PBS $(\mathrm{pH}=7.4)$ by probing its absorption and fluorescence (FL) spectra. As shown in Fig. 2B, IR-PEG-FA possessed a broad absorption band with two peaks at $810 \mathrm{~nm}$ and $1000 \mathrm{~nm}$. The mass extinction coefficient of IR-PEG-FA at $808 \mathrm{~nm}$ were $58.65 \mathrm{mg}^{-1} \mathrm{~cm}^{-1} \mathrm{~mL}$ (Fig. 2C), which was superior to many reported NIR absorption materials, such as carbon nanotubes $\left(46.5 \mathrm{mg}^{-1} \mathrm{~cm}^{-1} \mathrm{~mL}\right)$, gold nanorods (13.89 $\mathrm{mg}^{-1} \mathrm{~cm}^{-1} \mathrm{~mL}$ ) and conjugated polymers (55 $\left.\mathrm{mg}^{-1} \mathrm{~cm}^{-1} \mathrm{~mL}\right) .{ }^{47}$ Besides, the fluorescence spectrum of IRPEG-FA was investigated and it showed an evident NIR-II emission property with a peak at around $1100 \mathrm{~nm}$ at an
A
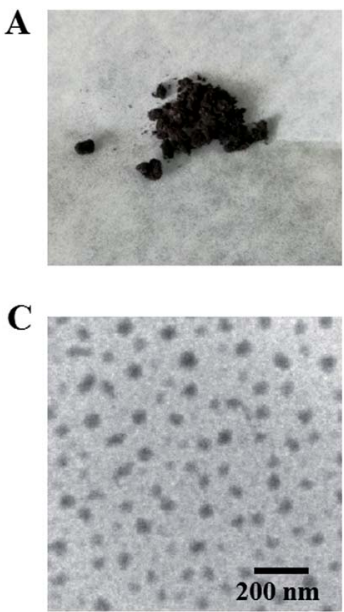

B
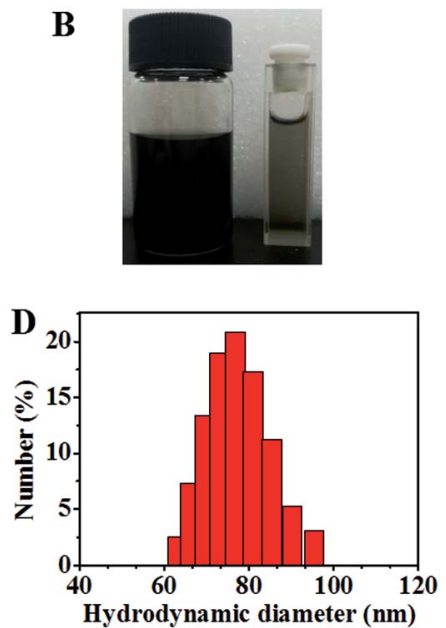

Fig. 1 Characterization of physical performances of IR-PEG-FA. (A) Picture of freeze-dried IR-PEG-FA. (B) Picture of IR-PEG-FA solution (left: $1 \mathrm{mg} \mathrm{mL}^{-1}$ and right: $0.05 \mathrm{mg} \mathrm{mL}^{-1}$ ) in PBS ( $\mathrm{pH}=7.4$ ). (C) TEM image of IR-PEG-FA. (D) DLS of IR-PEG-FA. 


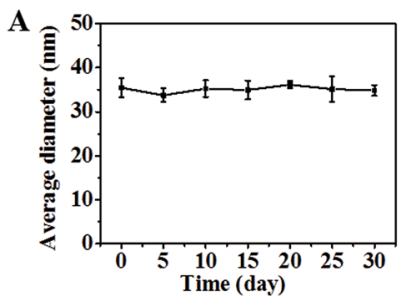

C

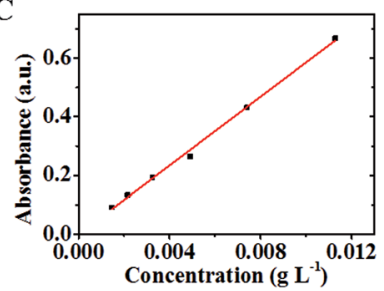

B

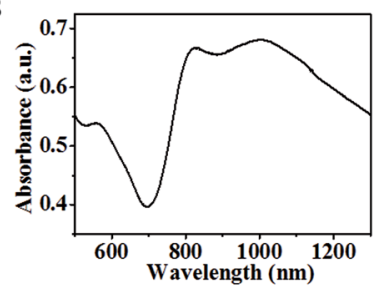

D

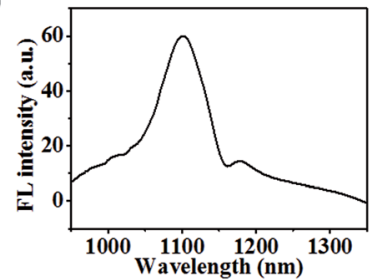

Fig. 2 In vitro characterization of IR-PEG-FA. (A) Hydrodynamic diameters of the IR-PEG-FA at different time points. (B) Absorption spectrum of IR-PEG-FA in PBS ( $\mathrm{pH}=7.4)$. (C) The plot of absorption density at $808 \mathrm{~nm}$ versus concentration. (D) Fluorescence spectrum of IR-PEG-FA in PBS ( $\mathrm{pH}=7.4)$

excitation wavelength of $808 \mathrm{~nm}$ (Fig. 2D). Herein, considering the maximum absorption of IR-PEG-FA and penetration depth for biological imaging in vivo, the $808 \mathrm{~nm}$ laser was used. ${ }^{32}$ Thus, the strong NIR absorption and NIR-II emission of IR-PEGFA could provide a theoretical basis for PAI and NIR-II imaging.

\section{In vitro PAI and NIR-II imaging}

To evaluate the PAI property, the PA signal intensities at $808 \mathrm{~nm}$ of IR-PEG-FA in aqueous solution were recorded. From Fig. 3A, the strong and bright PAI images indicated that IR-PAG-FA possesses excellent PA property and provided an opportunity for in vivo PAI. Besides, IR-PEG-FA exhibited a linear dependence between the sample concentrations and PA signals (Fig. 3B). Next, to further study the feasibility of NIR-II imaging, the fluorescence image in the NIR-II region of IR-PEG-FA was acquired at an excitation wavelength of $808 \mathrm{~nm}$. As shown in Fig. 3C, IR-PEG-FA displayed a significant NIR-II emission efficacy and possessed a strong NIR-II emission signal. Besides, to

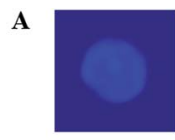

B

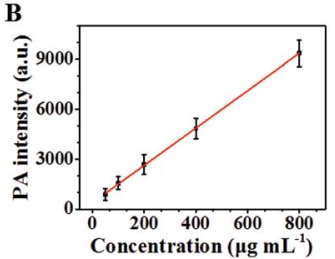

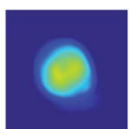

C

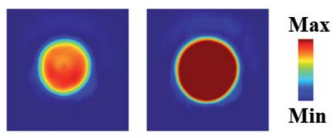

D

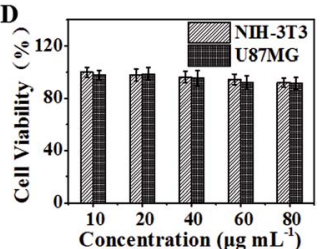

Fig. 3 In vitro dual-mode imaging and cell viability of IR-PEG-FA. (A) In vitro PAI of IR-PEG-FA with different concentrations $(0.05,0.1,0.2,0.4$ and $0.8 \mathrm{mg} \mathrm{mL}^{-1}$ ) in PBS ( $\left.\mathrm{pH}=7.4\right)$. (B) Linear dependence between concentrations and PA signals of IR-PEG-FA. (C) In vitro NIR-II imaging of IR-PEG-FA (0.4 mg mL ${ }^{-1}$ ) in PBS (pH = 7.4). (D) Cell viability of NIH3T3 and U87MG cells after cultivation with IR-PEG-FA at different concentrations.

explore the cytotoxicity of IR-PEG-FA, the cellular viabilities of NIH3T3 and U87MG cells after administration with IR-PEG-FA were evaluated, and Fig. 3D shows that the agent possessed fine cytocompatibility. All of these proved that IR-PEG-FA demonstrates incomparable advantages for further in vivo imaging applications.

\section{In vivo PAI and NIR-II imaging}

The capability of IR-PEG-FA for targeted in vivo PAI and NIR-II imaging was further proved using the U87MG tumor mouse model. As shown in Fig. 4A, before intravenous injection of IRPEG-FA via the tail, the tumor site exhibited low PA signals at $808 \mathrm{~nm}$ on account of the intrinsic background signal. ${ }^{49}$ Owing to the introduction of a molecular imaging ligand, along with the enhanced permeation and retention (EPR) effect of the pathological tumor sites, the IR-PEG-FA possessed more tumor-specific targeting performance for in vivo imaging. As we expected, a distinct and bright PA signal increase in the tumor regions could be detected after IR-PEG-FA injection. At $4 \mathrm{~h}$ post injection, the PA signal within the tumor achieved a maximum value, indicating the effective accumulation of IRPEG-FA, owing to both active and passive tumor targeting. After that, the PA signal around the tumor area decreased gradually because of the biological metabolism and biodegradability of the NPs in the physiological environment in vivo. Besides, at $24 \mathrm{~h}$ post injection of IR-PEG-FA, the mice were euthanized. The major organs including the lungs, liver, heart, kidney, spleen, and tumor were excised and imaged via PAI (Fig. 5A). The liver and spleen tissues exhibited bright and strong PA intensity, whereas the heart, lung, kidney and tumor tissues possessed relatively low signals (Fig. 5B). Thus, this
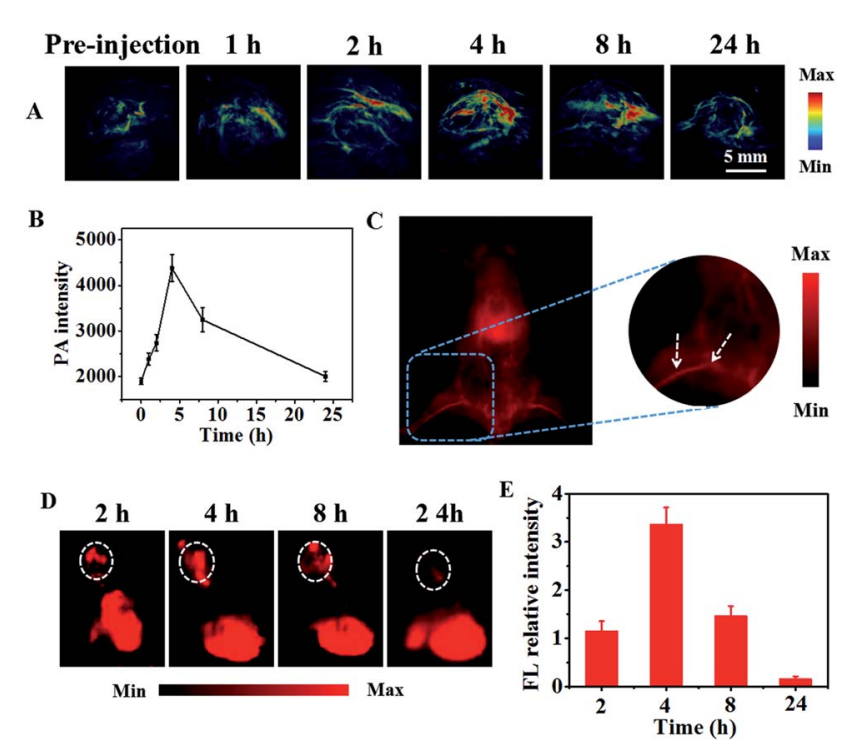

Fig. 4 In vivo PAl and NIR-II imaging. (A) Tumor mice for in vivo PAI at varying time points after systemic administration with IR-PEG-FA. (B) The quantitative PA signals of tumor sites at different time points. (C) The whole-body NIR-II images treated with IR-PEG-FA at 2 min postinjection. (D) In vivo NIR-II imaging of tumor mice in a series of time points. (E) The relative NIR-II fluorescence signals of tumor sites. 
manifested that the administered IR-PEG-FA was completely cleared mainly through the hepatobiliary system.

To test the NIR-II imaging ability of IR-PEG-FA in vivo, the U87MG tumor-bearing mice were administrated with $150 \mu \mathrm{L}$ of NPs $\left(1 \mathrm{mg} \mathrm{mL}^{-1}\right)$ via tail vein. Interestingly, the blood vessels of the whole body could be distinctly detected at 2 min post injection using IR-PEG-FA (Fig. 4C) with high resolution and excellent image quality. Then, the fluorescence signals of tumor sites were gradually lightened along with the accumulation of IR-PEG-FA (Fig. 4D and E). At $4 \mathrm{~h}$, the fluorescence signal of the tumor region achieved the peak, which was consistent with that in PAI. Particularly, the maximum fluorescence signal intensity at $4 \mathrm{~h}$ post-injection was approximately 21 fold than that at $24 \mathrm{~h}$ post-injection with IR-PEG-FA, which further indicated the good tumor-specific targeting property of IR-PEG-FA and excellent biological biodegradable (Fig. 4E). These results indicated that IR-PEG-FA as a watersoluble and biocompatible NIR-II fluorescence agent fits biological imaging perfectly.

\section{Toxicity evaluation}

Furthermore, IR-PEG-FA toxicity was further investigated by collecting and analyzing the major stained organ slices from mice after 30 days of administration with IR-PEG-FA. As the same as the control groups (administration with PBS), no distinct pathological tissue, cell apoptosis, or necrosis were seen in the spleen, kidney, liver, heart, and lung after administration with the agent, which signified the absence of distinct side effects (Fig. 6), similar to the previously reported NIR-II fluorophores. ${ }^{46}$ Altogether, these satisfying results demonstrated that IR-PEG-FA can be adopted for NIR-II fluorescence and NIR-triggered PAI in living mice.

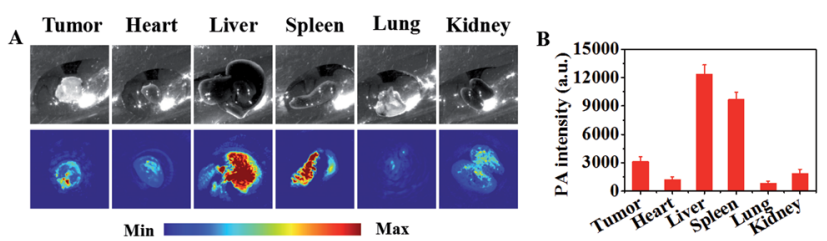

Fig. 5 Ex vivo PAl of major organs of mice after systemic administration of IR-PEG-FA at $24 \mathrm{~h}$. (A) Photographs (top) of the major organs and the PAl images (down) of them. (B) Quantitative PA intensities of major organs of mice at $24 \mathrm{~h}$ post-injection with IR-PEG-FA.

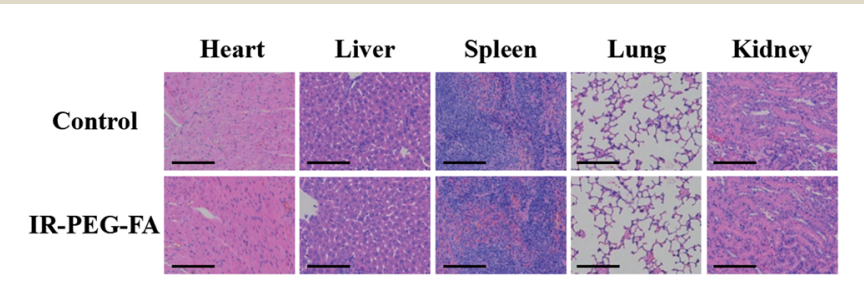

Fig. 6 Images of hematoxylin and eosin (H\&E) stained slices from major organs in mice after 30 days post-injection of IR-PEG-FA. Scale bar: $50 \mu \mathrm{m}$.

\section{Conclusions}

Summarily, we designed and synthesized a water-soluble, targeted and biocompatible dual-mode contrast agent that exhibited a strong NIR absorption and a bright fluorescence emission signal in the NIR-II region for in vivo PAI and NIR-II imaging. We believed that the tailor-made IR-PEG-FA for PAI and NIR-II fluorescence dual-modal imaging will capture widespread attention for the clinical applications of molecular imaging.

\section{Conflicts of interest}

There are no conflicts to declare.

\section{Acknowledgements}

The work was supported by the National Natural Science Foundation of China (81572109).

\section{References}

1 B. R. Smith and S. S. Gambhir, Chem. Rev., 2017, 117, 901986.

2 X. Li, J. Kim, J. Yoon and X. Chen, Adv. Mater., 2017, 29, 1606857.

3 Y. Lyu and K. Pu, Adv. Sci., 2017, 4, 1600481.

4 Y. Wang, K. Zhou, G. Huang, C. Hensley, X. Huang, X. Ma, T. Zhao, B. D. Sumer, R. J. DeBerardinis and J. Gao, Nat. Mater., 2014, 13, 204-212.

5 J. Kim, Y. Piao and T. Hyeon, Chem. Soc. Rev., 2009, 38, 372390.

6 E. A. Owens, M. Henary, G. El Fakhri and H. S. Choi, Acc. Chem. Res., 2016, 49, 1731-1740.

7 M. Elsabahy, G. S. Heo, S. M. Lim, G. Sun and K. L. Wooley, Chem. Rev., 2015, 115, 10967-11011.

8 D. E. Lee, H. Koo, I. C. Sun, J. H. Ryu, K. Kim and I. C. Kwon, Chem. Soc. Rev., 2012, 41, 2656-2672.

9 T. H. Shin, Y. Choi, S. Kim and J. Cheon, Chem. Soc. Rev., 2015, 44, 4501-4516.

10 B. E. Rolfe, I. Blakey, O. Squires, H. Peng, N. R. Boase, C. Alexander, P. G. Parsons, G. M. Boyle, A. K. Whittaker and K. J. Thurecht, J. Am. Chem. Soc., 2014, 136, 2413-2419.

11 Q. Fan, K. Cheng, X. Hu, X. Ma, R. Zhang, M. Yang, X. Lu, L. Xing, W. Huang, S. S. Gambhir and Z. Cheng, J. Am. Chem. Soc., 2014, 136, 15185-15194.

12 K. Yang, L. Hu, X. Ma, S. Ye, L. Cheng, X. Shi, C. Li, Y. Li and Z. Liu, Adv. Mater., 2012, 24, 1868-1872.

13 T. Liu, M. Zhang, W. Liu, X. Zeng, X. Song, X. Yang, X. Zhang and J. Feng, ACS Nano, 2018, 12, 3917-3927.

$14 \mathrm{~J}$. Lin, M. Wang, H. Hu, X. Yang, B. Wen, Z. Wang, O. Jacobson, J. Song, G. Zhang, G. Niu, P. Huang and X. Chen, Adv. Mater., 2016, 28, 3273-3279.

15 X. R. Song, X. Wang, S. X. Yu, J. Cao, S. H. Li, J. Li, G. Liu, H. H. Yang and X. Chen, Adv. Mater., 2015, 27, 3285-3291.

16 Y. Wang, T. Yang, H. Ke, A. Zhu, Y. Wang, J. Wang, J. Shen, G. Liu, C. Chen, Y. Zhao and H. Chen, Adv. Mater., 2015, 27, 3874-3882. 
17 X. Hu, F. Lu, L. Chen, Y. Tang, W. Hu, X. Lu, Y. Ji, Z. Yang, W. Zhang, C. Yin, W. Huang and Q. Fan, ACS Appl. Mater. Interfaces, 2017, 9, 30458-30469.

18 X. Wang, H. Hu, H. Zhang, C. Li, B. An and J. Dai, Nano Res., 2017, 11, 1069-1081.

19 R. Hu, Y. Wang, X. Liu, G. Lin, C. H. Tan, W.-C. Law, I. Roy and K.-T. Yong, RSC Adv., 2013, 3, 8495.

20 Y. Tian, Y. Zhao, W. Liu, Y. Liu, Y. Tang, Z. Teng, C. Zhang, S. Wang and G. Lu, RSC Adv., 2018, 8, 32200-32210.

21 K. Pu, A. J. Shuhendler, J. V. Jokerst, J. Mei, S. S. Gambhir, Z. Bao and J. Rao, Nat. Nanotechnol., 2014, 9, 233-239.

22 K. Pu, J. Mei, J. V. Jokerst, G. Hong, A. L. Antaris, N. Chattopadhyay, A. J. Shuhendler, T. Kurosawa, Y. Zhou, S. S. Gambhir, Z. Bao and J. Rao, Adv. Mater., 2015, 27, 5184-5190.

23 B. Guo, Z. Sheng, D. Hu, A. Li, S. Xu, P. N. Manghnani, C. Liu, L. Guo, H. Zheng and B. Liu, ACS Nano, 2017, 11, 1012410134.

24 J. Qi, Y. Fang, R. T. K. Kwok, X. Zhang, X. Hu, J. W. Y. Lam, D. Ding and B. Z. Tang, ACS Nano, 2017, 11, 7177-7188.

25 S. Zhang, W. Guo, J. Wei, C. Li, X. J. Liang and M. Yin, ACS Nano, 2017, 11, 3797-3805.

26 Q. Fan, K. Cheng, Z. Yang, R. Zhang, M. Yang, X. Hu, X. Ma, L. Bu, X. Lu, X. Xiong, W. Huang, H. Zhao and Z. Cheng, Adv. Mater., 2015, 27, 843-847.

27 C. Cui, Z. Yang, X. Hu, J. Wu, K. Shou, H. Ma, C. Jian, Y. Zhao, B. Qi, X. Hu, A. Yu and Q. Fan, ACS Nano, 2017, 11, 3298-3310.

28 C. Yin, Y. Tang, X. Li, Z. Yang, J. Li, X. Li, W. Huang and Q. Fan, Small, 2018, 14, 1703400.

29 Y. Jiang and K. Pu, Acc. Chem. Res., 2018, 51, 1840-1849.

30 A. L. Antaris, H. Chen, K. Cheng, Y. Sun, G. Hong, C. Qu, S. Diao, Z. Deng, X. Hu, B. Zhang, X. Zhang, O. K. Yaghi, Z. R. Alamparambil, X. Hong, Z. Cheng and H. Dai, Nat. Mater., 2016, 15, 235-242.

31 G. Hong, A. L. Antaris and H. Dai, Nat. Biomed. Eng., 2017, 1, 0010.

32 G. Hong, Y. Zou, A. L. Antaris, S. Diao, D. Wu, K. Cheng, X. Zhang, C. Chen, B. Liu, Y. He, J. Z. Wu, J. Yuan, B. Zhang, Z. Tao, C. Fukunaga and H. Dai, Nat. Commun., 2014, 5, 4206.

33 G. Hong, S. Diao, J. Chang, A. L. Antaris, C. Chen, B. Zhang, S. Zhao, D. N. Atochin, P. L. Huang, K. I. Andreasson, C. J. Kuo and H. Dai, Nat. Photonics, 2014, 8, 723-730.
34 J. Qi, C. Sun, A. Zebibula, H. Zhang, R. T. K. Kwok, X. Zhao, W. Xi, J. W. Y. Lam, J. Qian and B. Z. Tang, Adv. Mater., 2018, 30, 1706856.

35 Q. Yang, Z. Ma, H. Wang, B. Zhou, S. Zhu, Y. Zhong, J. Wang, H. Wan, A. Antaris, R. Ma, X. Zhang, J. Yang, X. Zhang, H. Sun, W. Liu, Y. Liang and H. Dai, Adv. Mater., 2017, 29, 1605497.

36 Q. Miao and K. Pu, Adv. Mater., 2018, e1801778, DOI: 10.1002/adma.201801778.

37 G. Hong, J. C. Lee, J. T. Robinson, U. Raaz, L. Xie, N. F. Huang, J. P. Cooke and H. Dai, Nat. Med., 2012, 18, 1841-1846.

38 K. Shou, Y. Tang, H. Chen, S. Chen, L. Zhang, A. Zhang, Q. Fan, A. Yu and Z. Cheng, Chem. Sci., 2018, 9, 3105-3110.

39 Y. Sun, C. Qu, H. Chen, M. He, C. Tang, K. Shou, S. Hong, M. Yang, Y. Jiang, B. Ding, Y. Xiao, L. Xing, X. Hong and Z. Cheng, Chem. Sci., 2016, 7, 6203-6207.

40 Q. Yang, Z. Hu, S. Zhu, R. Ma, H. Ma, Z. Ma, H. Wan, T. Zhu, Z. Jiang, W. Liu, L. Jiao, H. Sun, Y. Liang and H. Dai, J. Am. Chem. Soc., 2018, 140, 1715-1724.

41 K. Welsher, S. P. Sherlock and H. Dai, Proc. Natl. Acad. Sci. U. S. A., 2011, 108, 8943-8948.

42 A. L. Antaris, J. T. Robinson, O. K. Yaghi, G. Hong, S. Diao, R. Luong and H. Dai, ACS Nano, 2013, 7, 3644-3652.

43 C. Li, F. Li, Y. Zhang, W. Zhang, X. Zhang and Q. Wang, ACS Nano, 2015, 9, 12255-12263.

44 Y. Zhang, G. Hong, Y. Zhang, G. Chen, F. Li, H. Dai and Q. Wang, ACS Nano, 2012, 6, 3695-3702.

45 D. J. Naczynski, M. C. Tan, M. Zevon, B. Wall, J. Kohl, A. Kulesa, S. Chen, C. M. Roth, R. E. Riman and P. V. Moghe, Nat. Commun., 2013, 4, 2199.

46 Z. Tao, G. Hong, C. Shinji, C. Chen, S. Diao, A. L. Antaris, B. Zhang, Y. Zou and H. Dai, Angew. Chem., 2013, 125, 13240-13244.

47 Y. Tang, Y. Li, X. Hu, H. Zhao, Y. Ji, L. Chen, W. Hu, W. Zhang, X. Li, X. Lu, W. Huang and Q. Fan, Adv. Mater., 2018, e1801140, DOI: 10.1002/adma.201801140.

48 K. Cheng, H. Chen, C. H. Jenkins, G. Zhang, W. Zhao, Z. Zhang, F. Han, J. Fung, M. Yang, Y. Jiang, L. Xing and Z. Cheng, ACS Nano, 2017, 11, 12276-12291.

49 C. Yin, X. Zhen, H. Zhao, Y. Tang, Y. Ji, Y. Lyu, Q. Fan, W. Huang and K. Pu, ACS Appl. Mater. Interfaces, 2017, 9, 12332-12339. 